\title{
Temporal variations of low-order spherical harmonic representations of sunspot group patterns: Evidence for solar spin-orbit coupling
}

\begin{abstract}
D. Juckett ${ }^{\star}$
Barros Research Institute, 2430 College Rd., Holt, MI 48842, USA and Department of Chemistry, Michigan State University, East Lansing, MI 48842, USA

Received 28 May 2002 / Accepted 19 November 2002

Abstract. The spatial and temporal patterns of sunspot groups spanning 125 years are explored using surface spherical harmonic (SSH) analysis at a temporal resolution of one Carrington Rotation (CR). SSHs spanning order, $1 \leq m \leq 12$, and degree, $l$, satisfying $(l-m) \leq 12$ are calculated for each CR from 300 to 1950 and are expressed as amplitude and spatial phase for each CR. Linear combinations of harmonics (lcSSHS), grouped by constant $m$ and either even or odd $l$ values, generate sectoral constructs with maximal power within the sunspot latitude band. The temporal variations of the lcSSHs describe the variations in longitudinal distributions of sunspot groups. The frequency components of these variations are examined separately using singular spectrum, Fourier, and wavelet analyses. The spatial phases of the lcSSHs display long-term secular, retrograde motions with superimposed near-decadal torsional oscillations. Two of these oscillatory components appear identifiable. One is an artifact of the differential rotation of the photosphere and has an 11-yr period. The other is in near synchrony with the proposed $\mathrm{d} L / \mathrm{d} t$ series representing the torque on the Sun as it orbits the solar system barycenter. In contrast, each of the amplitude series for the lcSSHs follow an 11-yr solar cycle envelope but also contain a strong periodic component at 5.6 years and multiple components with periods between 15-30 years. Several of these oscillations are predictable from spin-orbit coupling considerations. At finer time scales ( $20 \mathrm{CR})$, there is also evidence for abrupt spatial phase shifts consistent with standing waves at a surface or interface. The conclusion is that SSH decomposition of sunspot patterns can be used to extract intensity and drift variations at several time scales. The intermediate time scale contains evidence that solar activity is modulated by the Sun's motion around the solar system barycenter.
\end{abstract}

Key words. Sun: sunspots - Sun: activity - Sun: oscillations - Sun: rotation

\section{Introduction}

Latitudinal sunspot behavior is well documented and reveals both the temporal progression of sunspots from high to low latitude (butterfly diagram) and the differential rotation of the solar outer layers. Evidence has also accumulated that there are longitudinal concentrations of sunspots, as well as horizontal repeating patterns in the large scale magnetic field, sometimes referred to as nests or active longitudes, e.g., (Bogart 1982; Uddin et al. 1991; Petrovay \& Abuzeid 1991; Simon \& Legrand 1992; Mikhailutsa \& Makarova 1994, 1999; Benevolenskaya et al. 1999; Erofeev 1999; Das \& Nag 1999; Rivin 1999; De Toma et al. 2000). Many of these studies show apparent multi-cycle persistence and longitudal stability in these features. Other studies have provided evidence for intermediate periodicities (e.g., 155 day repeating patterns) in the occurrence of various solar activity phenomena (Wolff 1983; Lean \& Brueckner 1989; Verma et al. 1992; Javaraiah \& Komm 1999), as well as cycles that have periods greater than 11 years in indicators of sunspots, differential rotation, and coronal emissions (e.g., Berger et al. 1990; Feynman \& Gabriel 1990;

\footnotetext{
^ e-mail: juckett@msu.edu
}

Javaraiah \& Gokhale 1995, 1997; Juckett 1998) . The combination of these analyses implies that latitudinal and longitudinal patterns change cyclically at both short and long time scales, but very little work has been published that combines spatial distributions with detailed temporal development.

A few spatial/temporal analyses have been reported, utilizing modified spherical harmonic decompositions of sunspot distributions (Stenflo \& Vogel 1986; Gokhale \& Javaraiah 1990; Gokhale et al. 1992; Gokhale \& Javaraiah 1995; Erofeev 2001) but they have been limited in scope. They have focused on axisymmetric modes $(m=0)$, which are devoid of longitudinal information, and have evaluated the harmonics only over the dimensions of latitude and time. Other studies have revealed sectoral quadrant patterns that change in time and exhibit possible meridional flow (Mikhailutsa 1995) but these have been limited to harmonic order of one or two and time resolution on the order of solar cycle length. Thus, a detailed analysis of longitudinal sunspot patterns as a function of time remains to be performed.

The goal of this report is to present the initial results of surface spherical harmonic decompositions of sunspot distributions, with emphases on the construction of sectoral 
harmonics and their oscillatory behavior over 125 years. The temporal variations in both spatial phase and SSH amplitude are examined at various time scales. In particular, the temporal variations in the spatial phases provide evidence for longterm torsional oscillations, suggesting the Sun is responding to torques associated with its motion around the solar system barycenter. Variations in SSH amplitudes yield several frequency components, spanning time scales both shorter and longer than the 11-yr solar cycle. The long-term oscillations match those observed in several solar, geomagnetic, and cosmic rays time series (Juckett 2001) while the rapid oscillations exhibit abrupt phase shifts suggestive of surface standing waves.

The presentation of results is as follows. In Sect. 3.1, examples of sunspot group patterns are presented separately for the latitude and longitude dimensions. These provide the rationale for SSH analysis. In Sect. 3.2, the clustering of sunspot groups is shown to be independent of group area, thus simplifying the SSH analysis. In Sect. 3.3, the results of SSH analysis are shown and examples are presented of the temporal patterns in the phase and amplitude series. The strong phase correlations among symmetric and anti-symmetric SSHs of the same order are demonstrated and the corresponding linear combinations (lcSSHs) are taken. The remainder of the report focuses on the symmetric lcSSHs. In Sect. 3.4, the spatial phase time series are examined to reveal drift rates linearly increasing with order, $m$. This relationship is used to combine the phase series for spectral analysis. The oscillatory components of the phase series are then compared to the planetary-induced torque, $\mathrm{d} L / \mathrm{d} t$, and to the differential rotation of the photosphere. In Sect. 3.5, the lcSSH amplitude time series are examined for their common and persistent oscillatory components. In addition to the 11-yr cycle, several other oscillations are identified.

\section{Methods}

\subsection{Data}

Data for sunspot groups were obtained from the National Geophysical Data Center (NGDC), Solar-Terrestrial Physics division. The sunspot group data for 1874 to 1981 were from Greenwich Observatory and data for 1981 to 1999 were tabulated for multiple observatories by the United States Air Force. The areas (in millionths of the solar hemisphere) reported for each sunspot group was extracted along with latitude and longitude information for each day that the group was observed. If the Carrington longitude was not available, it was calculated from the other available information (e.g., UT of observation, longitude East or West of central meridian, and UT of Carrington rotation onset). Area estimates from different observatories for the same sunspot groups, on identical days, were averaged together.

\subsection{Analysis}

For SSH analysis, the mean latitude and longitude position of each sunspot group was calculated for the days it was observed during a given CR (Carrington rotation). Each group was assigned a value of 1.0 at its mean position, regardless of the number of days observed in a CR. All of the unique sunspot groups occurring in a CR constituted a single time sample, forming a sunspot representation of the solar sphere similar to a synoptic map. Although short-lived sunspots occurring only on the backside are missed, the complete surface is sampled uniformly. A total of 26 rotations had no recorded sunspot occurrence and were excluded. To simplify some spectral analyses, the amplitudes at these 26 CRs were given values of zero and the spatial phase series were interpolated.

The real and imaginary coefficients for each of the surface spherical harmonics for each CR were determined by the product and integration method, for $1 \leq m \leq 12$, and degree, $l$, satisfying $(l-m) \leq 12$. Specifically, the spherical harmonic equation (as implemented in Mathematica ${ }^{\mathrm{TM}}$, Wolfram Research) was evaluated at the latitude, longitude point of each sunspot group in a given $\mathrm{CR}$ and then summed over all groups. Since the zonal modes $[l, 0]$ offered no longitudinal phase information, they were excluded from further analysis. The magnitudes and phase angles were obtained from the real and imaginary coefficients of each SSH at each CR. "Phase-walk" series were calculated by correcting for the discontinuity across the $0 \pi \mid 2 \pi$ boundary. The phase-walk series always assumed the smallest of the two phase separations between adjacent CRs. The numerical first derivative of the phase-walk series was obtained for each CR from the slope within a seven point, centered window. This is referred to as the phase-slope series.

Linear combinations of SSH time series (denoted lcSSH) were created by summing the real and imaginary components of the appropriate SSHs. The lcSSH series were smoothed using boxcar averaging, 3-CR wide prior to calculation of amplitude and phase at each CR. For spectral analyses, every third point of the smoothed series was sampled to reduce the series length to 550 points. This eliminated any frequency artifacts generated by the smoothing kernel.

Three spectral analysis techniques were used to estimate noise, determine persistent harmonic content, and examine the spectral properties of each solar cycle. To segregate possible noise power from signal, singular spectrum analysis (SSA) was used (Vautard et al. 1992). This method generates dataspecific eigenvectors and corresponding eigenvalues for the number of eigenmodes chosen. Probable noise components are detectable in the eigenvalue plots as a "plateau" of values at high eigenmode number. SSA was performed with 110 eigenmodes (imbedding dimension) for all of the lcSSHs. This value is one-fifth of the series length, as recommended (Vautard et al. 1992). In the SSH amplitude series, the first two eigenmodes were used to isolate the main envelope that follows the 11-yr solar cycle. In the lcSSH spatial phase-walk series, the first two eigenmodes were used, in combination with linear detrending, to isolate and remove the random-walk effect inherent in the calculation of the phase-walk series. Sums of principal components were used to generate reconstructed components, denoted RC. These were further analyzed with Fourier analysis to identify persistent harmonic content. Fourier spectra were always calculated on series padded with zeros out to 4096 points to facilitate accurate frequency identification of detected bands. To identify cycle-unique oscillations, wavelet analysis (Torrence \& Campo 1998) was used. 


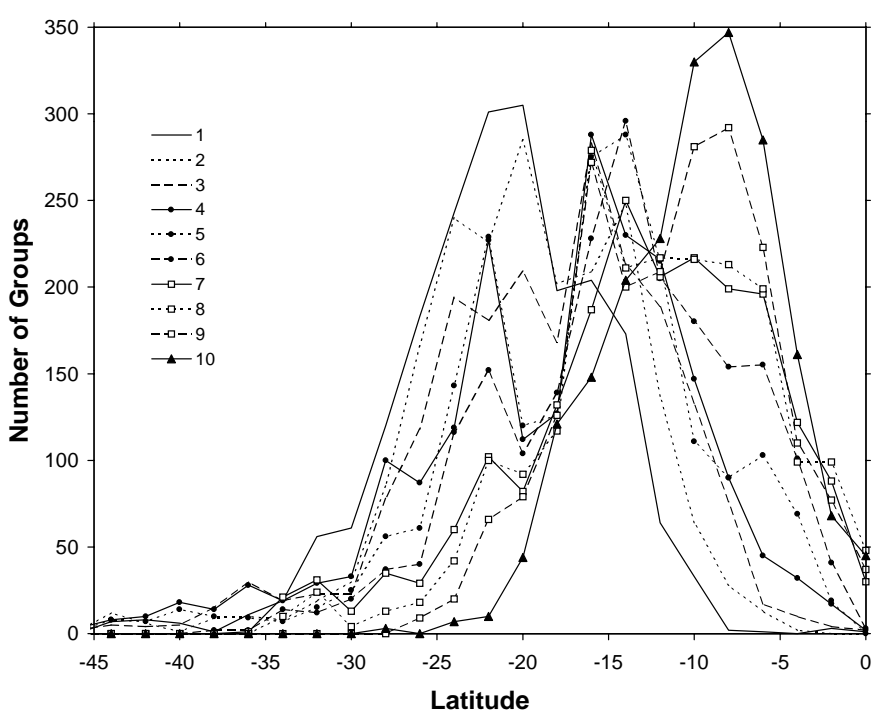

Fig. 1. Latitude distributions of equal sized groupings of sunspots during solar cycle 19. The sunspot groups for this cycle were sorted by hemisphere and ordered by time of occurrence. Each day a sunspot occurred was a separate entry in the list. A total of 11173 groups were in the data base for the southern hemisphere spanning the years 1955 to 1966 . Overlapping bins of 2000 groups were created, with an overlap of 1000 groups, and were distributed according to latitude in twodegree increments.

The Gaussian derivative wavelet (20th derivative) was used in complex form. Wavelet power, as a function of wavelet frequency and time, was calculated and presented as a 2diminsional "bubble" plot. All spectral analysis techniques were used as implemented in AutoSignal ${ }^{\mathrm{TM}}$ (SPSS inc.).

\section{Results}

\subsection{Generalized latitude and longitude patterns of sunspot groups}

Examples of sunspot group distributions, in the single dimensions of latitude and longitude, are shown in Figs. 1 and 2, respectively. In Fig. 1, the frequency histograms of overlapping subsets of 2000 sequentially observed sunspot groups are shown for the Southern hemisphere during solar cycle 19. This family of curves highlights the transition from high latitude predominance to low, but it also exhibits three latitude regions of high sunspot likelihood and two regions where cross-over clusters tend to occur, near -12 and -18 degrees. These points can be interpreted as transitions from one most likely latitude to another. Solar cycle 19 exhibits this effect most strongly, probably because it contains the greatest number of sunspots for construction of such curves, but all cycles 16-22 show similar behavior.

In Fig. 2, a generalized view of longitudinal clustering is shown for all sunspot groups documented between CR300 and CR1950. This two-state representation highlights the patterning that occurs at low spatial frequencies. Two general observations can be made. The first is the tendency for alternating spatial phase occurring every $50-100$ CRs. The second is the

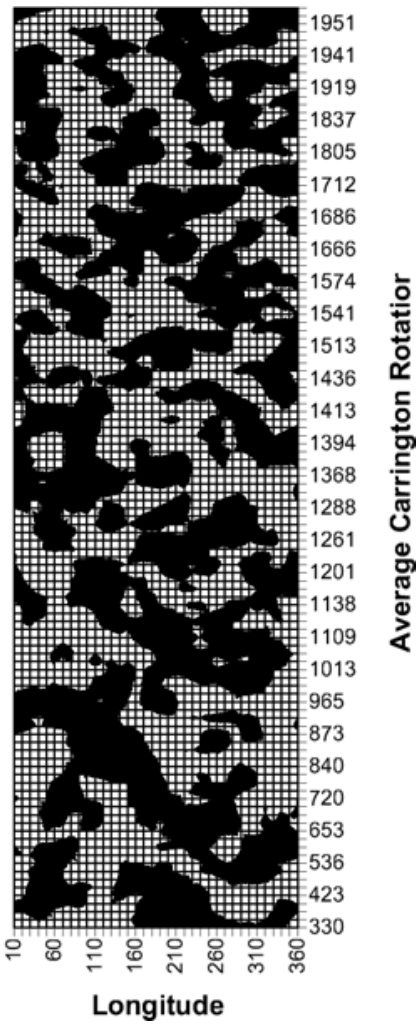

Fig. 2. Longitude distribution map for equal size groupings of northern hemisphere sunspots from CR300 - CR1950. Each horizontal line in the map represents the longitudinal distribution of 1000 sunspot groups taken sequentially in time, with no overlap, from 115407 sunspot entries of the northern hemisphere. The map was smoothed once by averaging a target point with all its nearest neighbors. The map is plotted as a filled contour graph with black regions representing prevalence greater than the mean value for the entire map, while unfilled areas are below the mean. The mean Carrington rotation for each grouping is indicated on the ordinate, which does not represent linear time.

apparent drift of spatial phase to lower longitudes (retrograde) as average $\mathrm{CR}$ increases.

Figures 1 and 2 represent two different time scales, but both show the general tendency of sunspots to cluster in different regions on the solar globe at different times. To obtain a better understanding of this behavior, surface spherical harmonics are used to simultaneously decompose both dimensions into orthogonal representations. The time courses of these representations can then be explored.

\subsection{Sunspot group area and clustering}

A simple cluster analysis was performed to ascertain if sunspot area needed to be considered during the decomposition of sunspot patterns. A cluster statistic, $S_{i}$, was defined as the sum of all inverse great circle distances (in degrees of arc) between a given sunspot group, $i$, and the remaining groups in the same latitudinal hemisphere during that CR. A large value of $S$ indicates that a given sunspot group is in close proximity to a significant proportion of the other sunspot groups in that CR. 


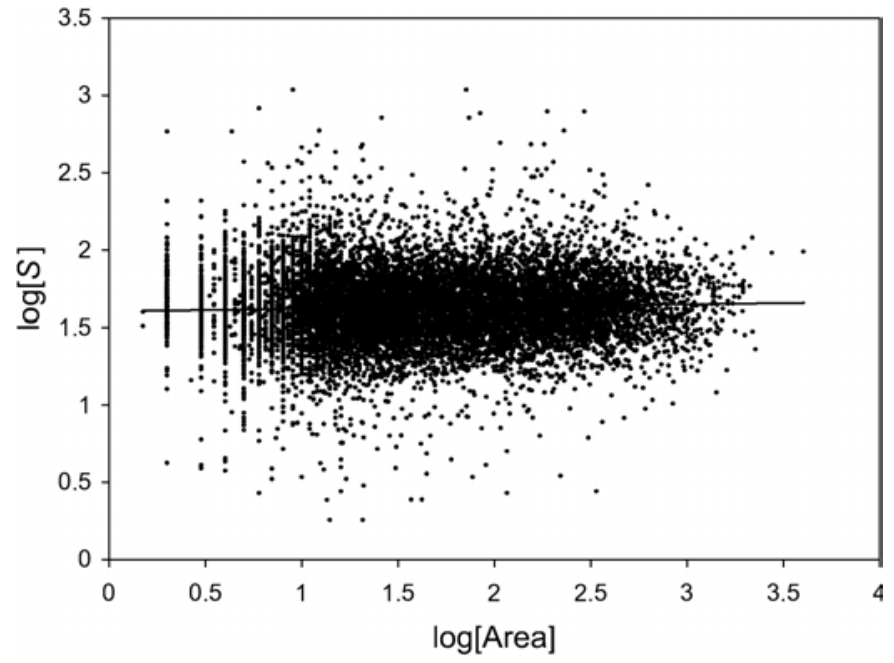

Fig. 3. The cluster statistic, $S$, for each sunspot group plotted versus sunspot group area for CRs 1300 to 1600 . A log-versus-log format (base 10) is used for plotting convenience. The horizontal line through the data represents a least-squares regression line, with slope of 0.015 .

The value of $S$ was calculated for each sunspot group in CR1300 through CR1600 and the full set of values is plotted versus group area in Fig. 3. The regression line and the distribution of points indicate that the cluster statistic, $S$, does not depend on sunspot area. From this it is concluded that any spatial patterning of sunspot groups is independent of sunspot size and, hence, sunspot position is sufficient to describe sunspot spatial patterns. The same lack of regression occurs when only a few CRs are examined (not shown) and is also true for all portions of a single sunspot cycle.

\subsection{Surface spherical harmonic analysis and construction of IcSSHs}

Surface spherical harmonics were calculated as described in Methods for each CR. The ranges of degree and order were chosen to provide an equal number of Associated Legendre Polynomials in the latitude dimension for each value of $m$, resulting in similar descriptive power for features in that dimension. Since sunspot groups are confined to relatively narrow latitude bands, a suitable number of SSHs are required to construct representations with power concentrated in the active latitudes. Twelve polynomials are capable of confining power as narrowly as 10 degrees full width at half maximum. This was deemed sufficient to allow construction of linear combinations of constant $m$ values, which could be used to decompose the low-order longitudinal patterns.

The temporal development of amplitude and spatial phase for a single SSH component is shown in Fig. 4. The smoothed Wolf sunspot series is shown in panel (a) for reference. The phase plots were repeated over three ranges of $2 \pi$ to highlight both the slow and rapid spatial phase changes. In panel (c), a continuous phase-walk series was calculated using an algorithm that eliminates phase jumps greater than $\pi$ between neighboring CRs caused by the discontinuity at the $0 \pi \mid 2 \pi$ interface.
The amplitude series contains high frequency variation that appears to be modulated by an envelope that follows the 11-yr sunspot cycle. The spatial phases exhibit a visually detectable drift, identifiable as broad horizontal stripes with a positive slope. The phase series also exhibits abrupt phase shifts, on the order of $\pi$, consistent with the high frequency variation in the amplitude series. Although not shown in detail, these shifts tend to occur at amplitude minima. The presence of the abrupt phase shifts causes a natural scatter in the series. Together with the unknown level of noise variation, it is difficult to establish an unbiased representation of the long-term variation in the spatial phase. The phase-walk series in panel (c) is a biased representation of phase changes because the smallest phase transitions are assumed at each CR. Therefore, it contains both the true phase drift trajectory and a random walk integration of the noise. When several such series are used in comparisons and in averages, however, the noise components are reduced and these series become useful.

To focus on the temporal variations in longitudinal sunspot patterns it is desirable to create linear combinations of SSHs that share common values of order, $m$, thus forming sectoral lcSSHs. This can only be justified if the variations in spatial phases are similar for the those SSHs being summed. Variational similarity was evaluated by comparing phase changes between pairs of SSHs using the first derivative of the phase-walk series (denoted phase-slope series). The strengths of the correlations among all combinations of these phase-slope series were calculated. Four examples from the 12090 comparisons are shown in Fig. 5. Diagonal clustering with a positive slope indicates high similarity between SSHs. The clustering in the negative slope correlates with the noise difference in the two series. The overall results indicate that strong correlations, with low noise differences, exist for those SSHs sharing the same $m$ value and having $l$ values either all odd or all even. Figure 6 shows this relationship. This defines two groupings for each $m$ value, one symmetric across the equator and the other anti-symmetric. Linear combinations were computed (see Methods) for each $m$ value and are denoted as either $m N e$ or $m N o$, where $N$ is an integer representing the value of $m$, " $e$ " indicates the sum over even $l$ values, and " $o$ " the sum over odd values. The set of symmetric lcSSHs is composed of $[m 1 o, m 2 e, m 3 o, m 4 e, \ldots]$, while the anti-symmetric subset consists of $[m 1 e, m 2 o, m 3 e, m 4 o, \ldots]$. These lcSSHs represent sectoral decompositions of the solar surface, which embody the first twelve longitudinal harmonics $(1 \leq m \leq 12)$ of sunspot distributions.

The amplitudes and spatial phases were calculated for each lcSSH after a 3 point smooth was applied. The symmetric subset comprised approximately two-thirds of the total power and is the focus of time series analyses presented in the next two sections.

\subsection{Temporal variations in symmetric IcSSH spatial phase drifts}

The temporal changes in the spatial phases contain several types of information that cannot be obtained from amplitude 

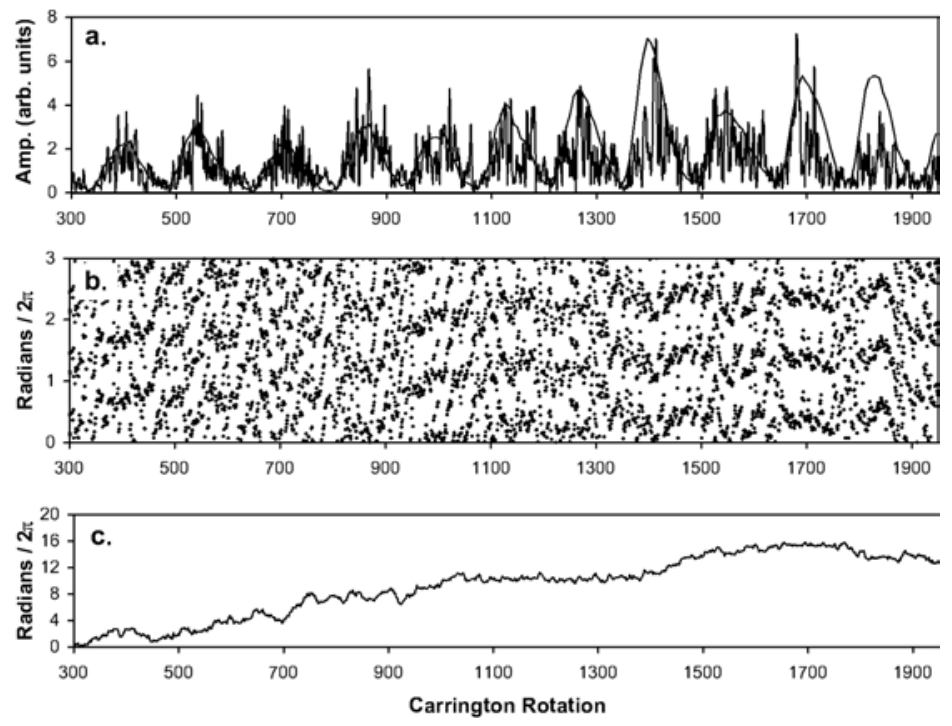

Fig. 4. Amplitude, spatial phase, and phase-walk time series for SSH $[2,2]$. a) The amplitude series was calculated after a threepoint smooth was applied to the complex coefficients of the SSH series. The smoothed monthly Wolf sunspot number is shown for reference. Scaling was arbitrarily chosen. b) The corresponding spatial phase series, for the smoothed SSH series, is repeated over three ranges of $2 \pi$. c) The phase-walk series was calculated from the spatial phase, as described in Methods.
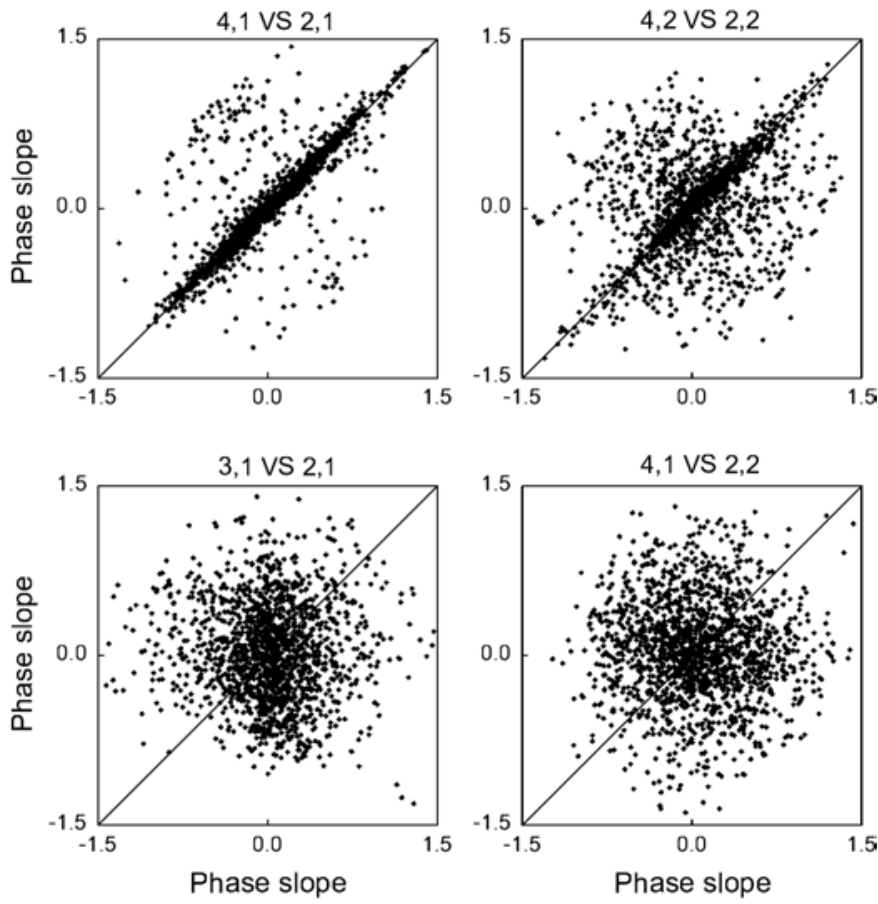

Fig. 5. Examples of phase slope scatter plots comparing four combinations of SSHs. The phase slopes were calculated from the phase-walk series using numerical differentiation on moving 7-point windows. The diagonal identity line is shown to allow qualitative comparison of each pair of SSH. A quantitative measure of correlation along the $y=x$ line was obtained by first calculating the minimal distance between each point and the line, then calculating the median of this set of values. The inverse of this median value was considered an estimate of diagonal correlation. The degree and order are denoted as $l, m$ and indicated above each panel.

alone or from spatially integrated series, such as the Wolf sunspot index. In particular, the movement of regions of high sunspot likelihood can be monitored over time scales spanning a few CRs to several hundred. In this section, the short and very long time scales will be examined qualitatively, followed by a quantitative extraction of the intermediate time scale variations for spectral analysis.

The abrupt phase shifts observed in the single SSHs (e.g., Fig. 4) also exist in the lcSSHs. A portion of the CR domain (cycle 19) is shown in Fig. 7 for three lcSSHs using a plotting technique that assigns each spatial phase point with an area proportional to the amplitude at that CR. All three lcSSHs show a strong oscillatory set of phase shifts during the time of solar cycle 19 maximum (near year 1958). During the solar cycle minimum, near 1963, some phase oscillations are observed but there is also a major retrograde drift (increase in phase). The regular oscillations occurring during solar maximum may indicate some type of standing wave that generates the release of sunspots, while the rapid phase drifts at cycle minimum may be simply due to the differential rotation of high-latitude sunspots occurring at the beginning of the next cycle. These two types of behavior occur at many maxima and minima throughout the 125 years of the dataset. This can be seen more clearly in Fig. 8, where six lcSSHs are shown over the full 125-yr interval using a 15-CR smoothing to highlight the long-term trends.

The long-term spatial phase trends in Fig. 8 are readily visible although not easily quantifiable. By viewing Fig. 8 at various angles, tilts, and distances, slopes that tend to increase with $m$ are clearly visible. Since the spatial phases are defined with respect to each harmonic's wavelength, drifts of features on the solar surface cause spatial phases to scale with order, $m$. These slopes persist for the full 125 years in most cases, although they sometimes exhibit abrupt shifts of $\pi$ radians. This persistence suggests long-term stability in some mechanisms responsible for sunspot genesis. The occasional phase inversions do not occur at the same times for each lcSSH suggesting that the harmonics are responding independently to the forces determining sunspot location. These features highlight the wealth of information that appears to reside in these phase changes. In this report, however, only a limited analysis can be presented.

An identifiable oscillation appears extractable from the intermediate time variations, so this is presented in detail. The phase-walk series were calculated for each of the lcSSHs (those with 3 CR smoothing) up through $m=12$. They resemble the 


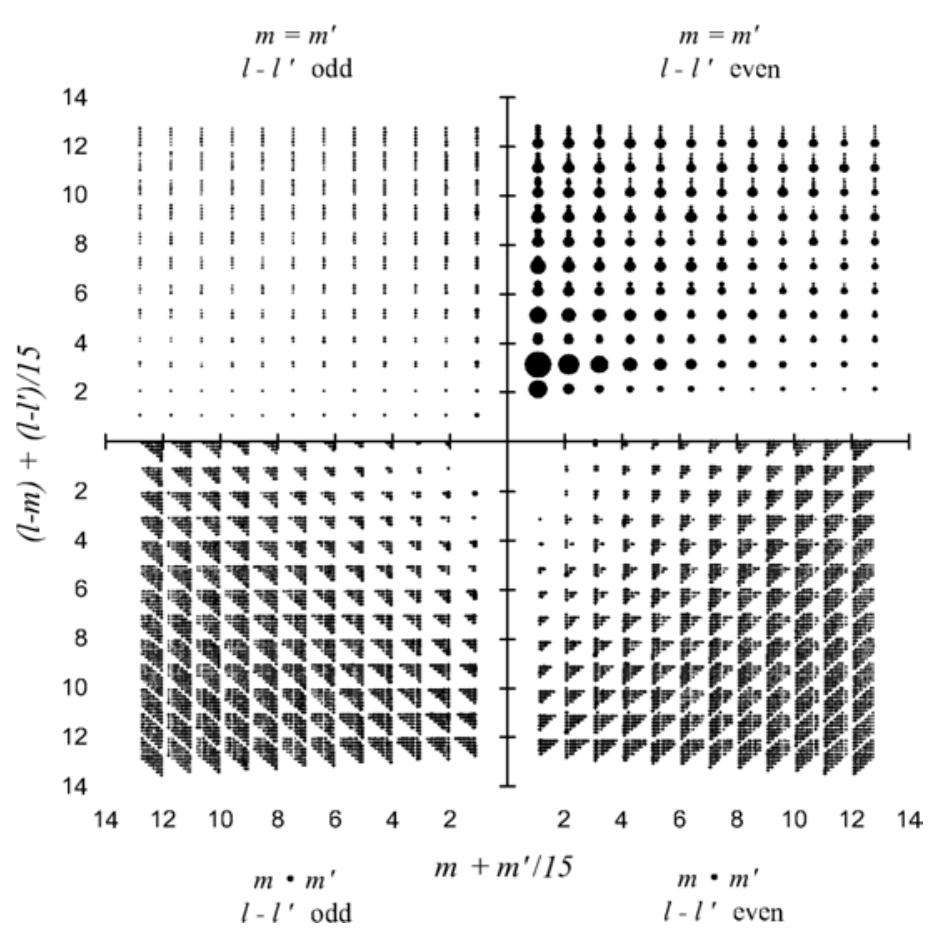

Fig. 6. Bubble plot of phase-slope correlations between all possible pairs of SSHs. The plot is divided into quadrants to sort the results according to comparisons of $m=m^{\prime}$ (upper half) or $m \neq m^{\prime}$ (lower half) and $\left(l-l^{\prime}\right)$ even (right half) or odd (left half). To accomodate all the different combinations, the ordinate and abscissa integral divisions are subdivided into 15 units to represent the various $\left(l^{\prime}-m^{\prime}\right)$ and $m^{\prime}$ values, respectively. The axes values for each quadrant begin in the center and increase toward the periphery i.e., there is no negative range. The degree of correlation (see main text) is proportional to spot area. Only the upper right quadrant contains correlations significantly above background.
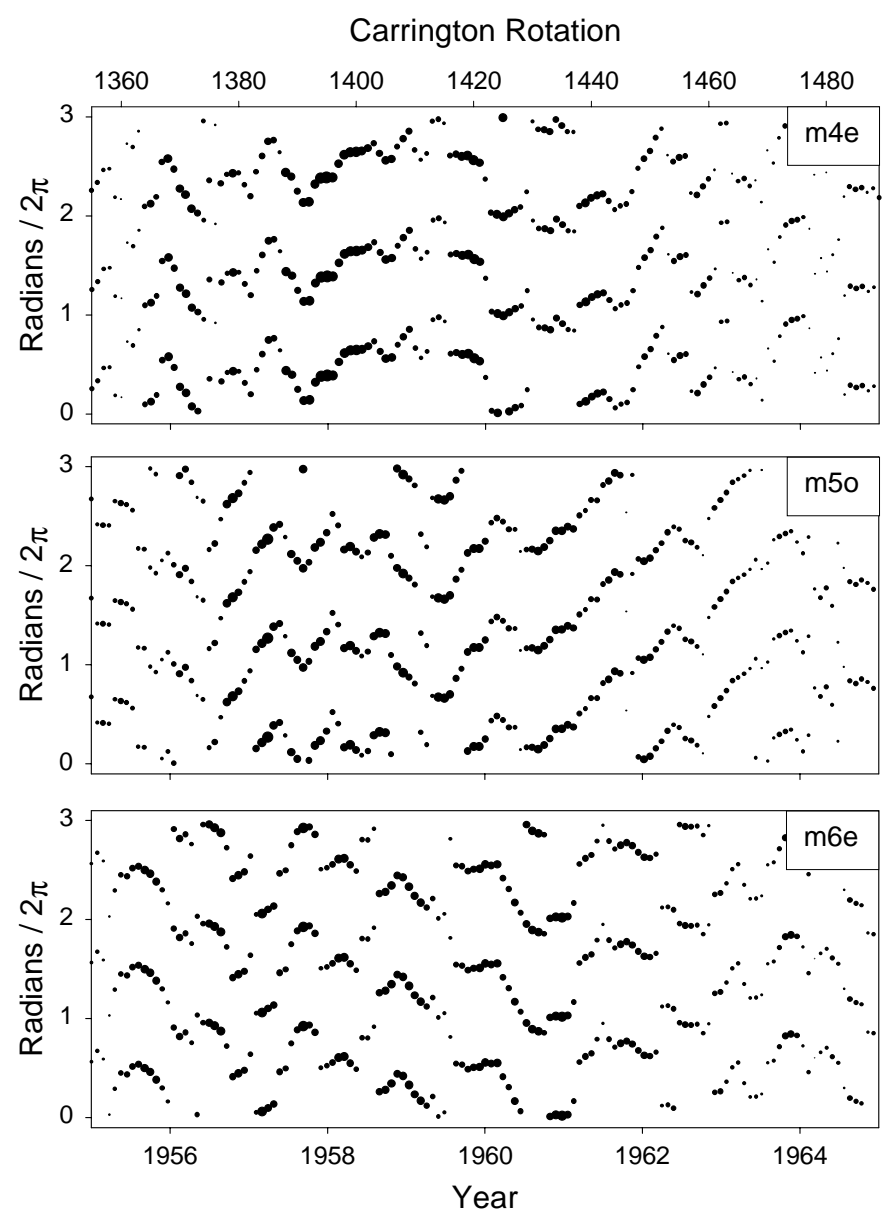

Fig. 7. Three examples of portions of lcSSH spatial phase series. This CR region encompasses most of solar cycle 19. Each series is repeated over three ranges of $2 \pi$ to highlight drift tendencies. Each point is weighted by area (bubble plot) according to the amplitude value of that $\mathrm{lcSSH}$ at that $\mathrm{CR}$. phase-walk series in Fig. 4. The symmetric set was averaged into a single series after dividing each series by $m$ to convert them to radians of solar rotation. Each series in the average was also weighted by the mean of the corresponding lcSSH amplitude series. It was anticipated that this series would contain the general prograde and retrograde variations in sunspot patterns that influence all the spatial harmonics. The linear trend was subtracted and the resultant series was decomposed using SSA with an imbedding dimension of 110 (see Fig. 9). The averaging procedure, combined with the linear detrending removed most of the random walk generated by noise. The remainder of the noise was identified in the SSA eigenmode spectrum of Fig. $9 \mathrm{~b}$ as the plateau of values at index values $15-110$. The first two eigenmodes are associated with secular, non-harmonic variations and are not considered further. The reconstructed eigenvectors 3-14 (Fig. 9c) were chosen for additional analysis. Fourier transform analysis of RC3-14 is shown in Fig. 9d. Two frequency domains of interest are identified as A and B. Region B is clearly the 11-yr sunspot cycle. Region A, however, contains cycles with periods of 20 to 13 years.

The time series for region A was reconstructed from the Fourier coefficients. This is shown in Fig. 10a. For comparison, the $\mathrm{d} L / \mathrm{d} t$ series of Jose (1965) is also shown, representing the anticipated orbital torque on the Sun by the giant planets. The visual similarity of these two series is quite remarkable. Comparison of the spectra (Fig. 10b) reveals the major overlap of the 19.6-yr cycle in both series. This is not a cycle that is seen in the amplitudes of solar processes or the many surrogates of solar activity (Juckett 2001). It is clearly distinct from both the 22.5-yr Hale cycle and the 17.7-yr cycle observed in these surrogates.

To further compare the series in Fig. 10a, the cross correlation was calculated and is shown in Fig. 10c. This is a routine method for determining the similarity between two 

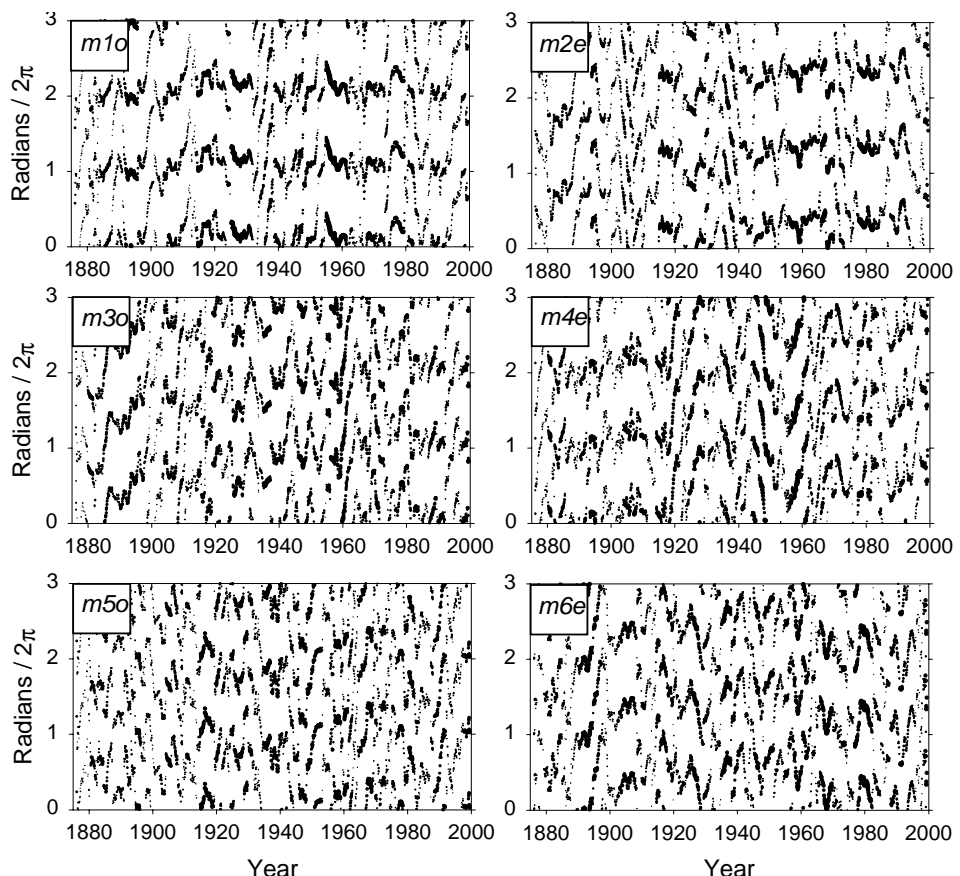

Fig. 8. First six lcSSH spatial phase series after $15 \mathrm{CR}$ smoothing. As before, smoothing occurred on the complex coefficients of the lcSSHs and then the spatial phase was calculated. Viewing this figure from different angles facilitates recognition of $m$-dependent drifts.
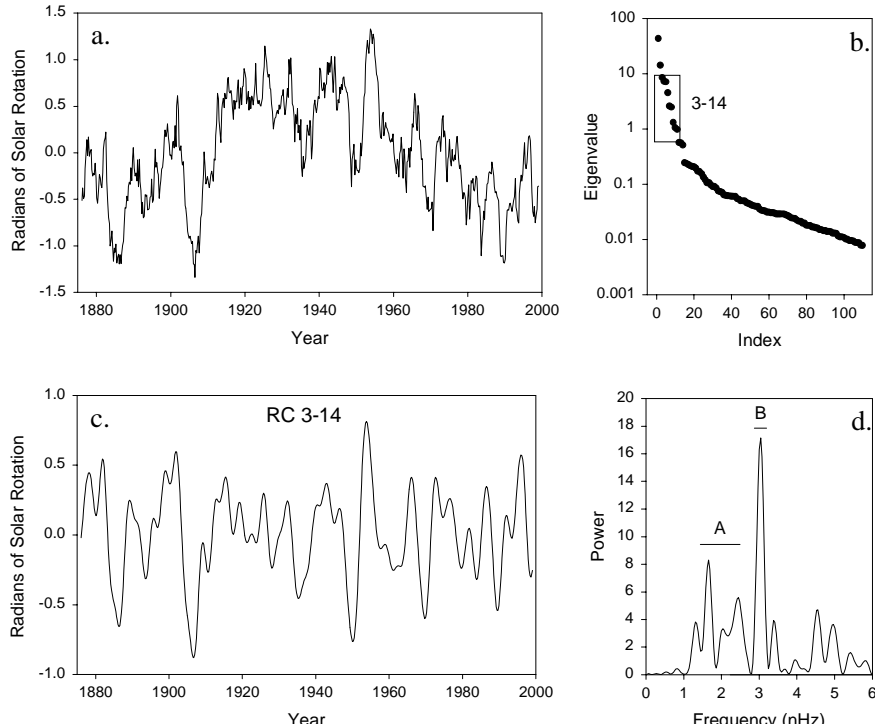

Fig. 9. Average phase-walk series from symmetric lcSSHs and selected spectral analysis. a) Sum of phase-walk series for the symmetric lcSSHs $m 1 o$ through $m 12 e$, after dividing each by $m$ and first-order detrending. b) SSA eigenvalue plot, with transition region values 3-14 denoted by the box. c) The reconstructed series using eigenvalues and eigenfunctions 3-14. d) Fourier transform of RC3-14 with identification of two frequency domains $\mathrm{A}$ and $\mathrm{B}$ that will be analyzed further.

signals or for identifying their commonly shared components. Positive correlation near zero lag reaches a value of approximately 0.7 indicating strong similarity and near synchrony. The Fourier spectra of both the cross correlation and $\mathrm{d} L / \mathrm{d} t$ are shown in Fig. 10d revealing that the two main frequencies of $\mathrm{d} L / \mathrm{d} t$ appear in region $\mathrm{A}$. When the waveforms for these cycles are extracted from $A$ and $\mathrm{d} L / \mathrm{d} t$, they are both nearly synchronous (Figs. 10e-f). Each of these pairs differ in phase by less than $10 \%$. The random probability of two components of two arbitrary series being simultaneously synchronous, to within $10 \%$ phase difference, is $0.1 \times 0.1$. Thus, the probability is at least 0.99 that $\mathrm{d} L / \mathrm{d} t$ and region A are related.

The other major component in the RC3-14 series of Fig. 9c is the single peak of region $\mathrm{B}$. This may be an artifact of the differential rotation of the high latitudes. The time series for region $\mathrm{B}$ was reconstructed from the Fourier coefficients and is shown in Fig. 11 together with the Wolf sunspot series. When region $\mathrm{B}$ exhibits high positive values, it is near solar minima or at the beginning of a cycle. Since positive values indicate retrograde motion of the spherical harmonics, this is consistent with the retrograde motion of the high latitude sunspots in the early part of the solar cycle (Howard 1996).

The conclusion drawn from the analysis of the intermediate variations contained in the average spatial phase series is that two main frequency components are common across all SSHs. One of these is an 11-yr retrograde-prograde oscillation derived from the average latitude of sunspot activity moving across the differential rotation of the photosphere. The other is a slower cycle, dominated by a 19.6-yr retrograde-prograde oscillation, which correlates with an orbital torque function of the Sun. This may be evidence for spin-orbit coupling between the orbital torque and torsional rotations.

\subsection{Temporal variations in IcSSH amplitude series}

The amplitude series of the lcSSHs exhibit several oscillations over a variety of time scales. To distinguish between signal and noise, the symmetric lcSSHs were examined by various spectral analysis techniques. In Fig. 12, the amplitude series for the $m 4 e$ lcSSHs is shown together with the main harmonic component (RC1-2) and the residual component (RC3-110) obtained by SSA. This was repeated for each symmetric lcSSH spanning $m 1 o$ to $m 12 e$. The dominant RC1-2 component represents the 11-yr solar cycle and is common in all lcSSHs. Other 

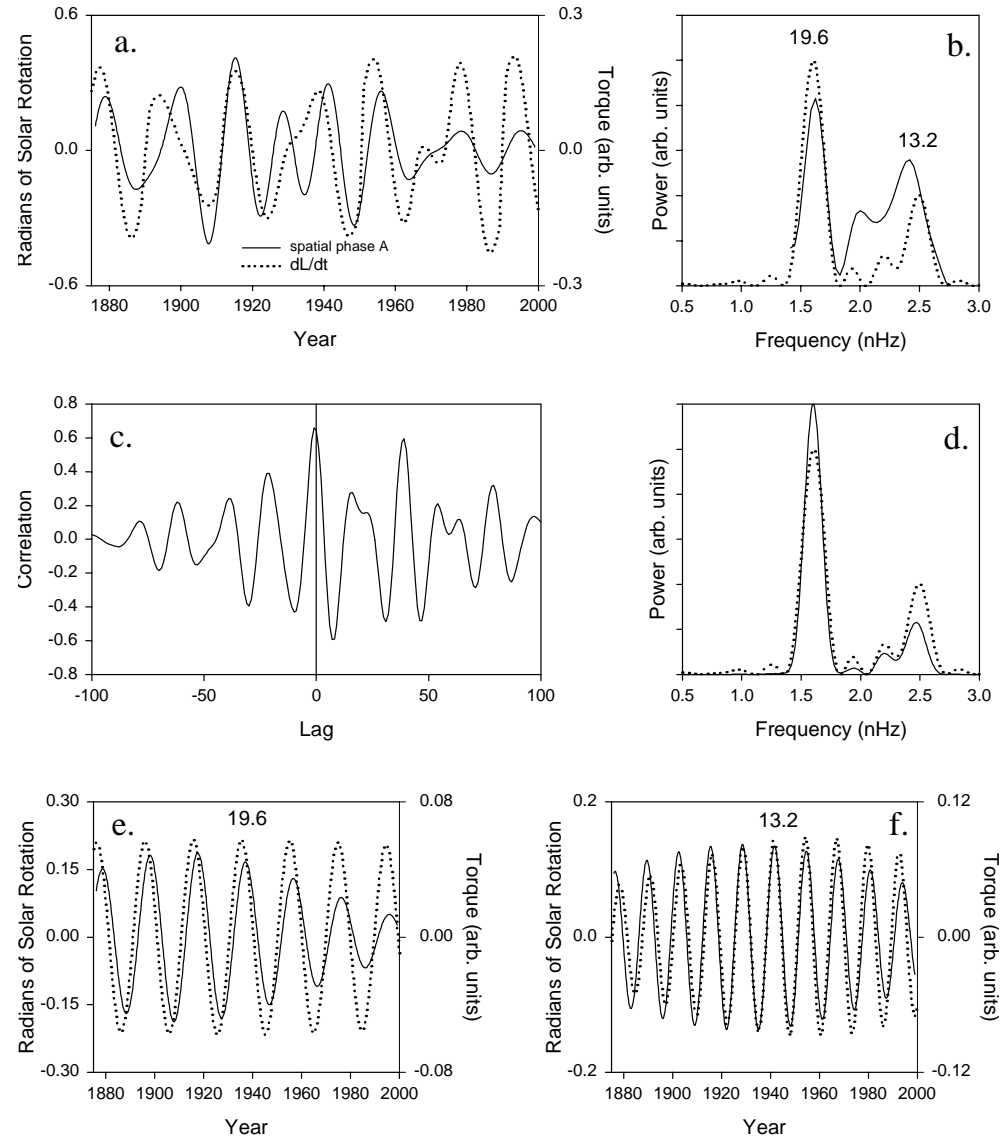

Year

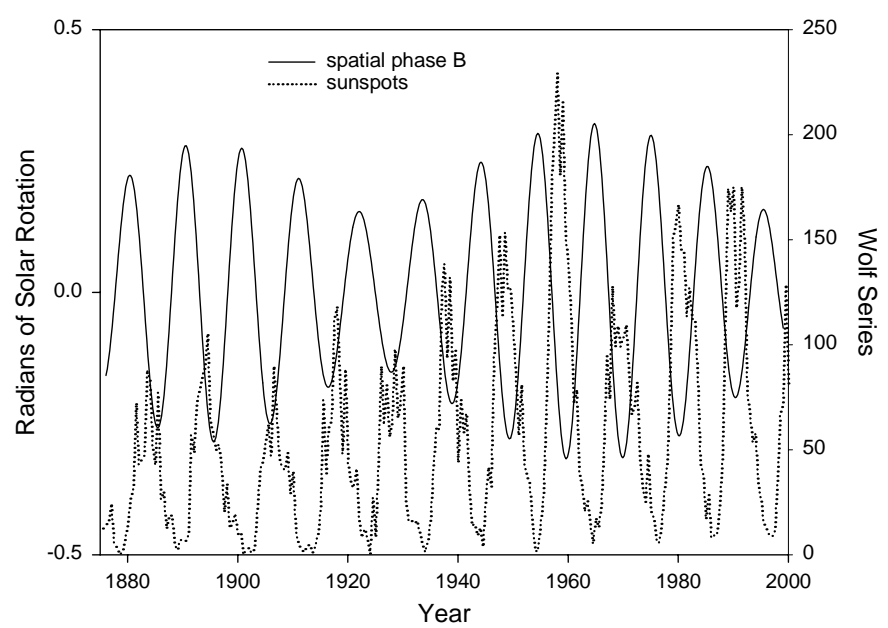

Fig. 11. Comparison of region B (11yr cycle) to Wolf Sunspot series. The series for region B of RC3-14 (Fig. 9) was reconstructed from the Fourier coefficients near $3 \mathrm{nHz}$ The Wolf series is the unsmoothed monthly count.

regions of both higher and lower frequency show consistent overlap up to approximately $6 \mathrm{nHz}$, representing common oscillations in most or all lcSSHs (Fig. 13a). As seen in the Fig. 13b, the Fourier spectrum of the sum of all 12 RC3-110 series highlight these common frequencies along with a dramatic reduction in power above $6 \mathrm{nHz}$.

For the summation series to generate peaks of significant power in the Fourier spectrum, the corresponding oscillations
Fig. 10. Various analyses of region A from RC3-14 of Fig. 9. a) Comparison of region $A$ and the $d L / d t$ series of Jose (1965). The series for region A was reconstructed from the Fourier coefficients, interpolated with a cubic spline, and sampled yearly to make it compatible with the $\mathrm{d} L / \mathrm{d} t$ series. The legend denotes the line types used for panels (a), (b), (e), and (f). b) Fourier spectra of the two series in panel (a). c) Cross-correlation of the two series in panel (a), truncated at lag $+-100 \mathrm{yr}$. A negative lag indicates a negative time shift for region A with respect to $\mathrm{d} L / \mathrm{d} t$. d) Fourier spectra of the cross-correlation (solid) and $\mathrm{d} L / \mathrm{d} t$ (dotted). e) Waveforms, reconstructed from Fourier components for $19.6 \mathrm{yr}$ components of the two series in panel (a) and, f) the 13.2 yr component.

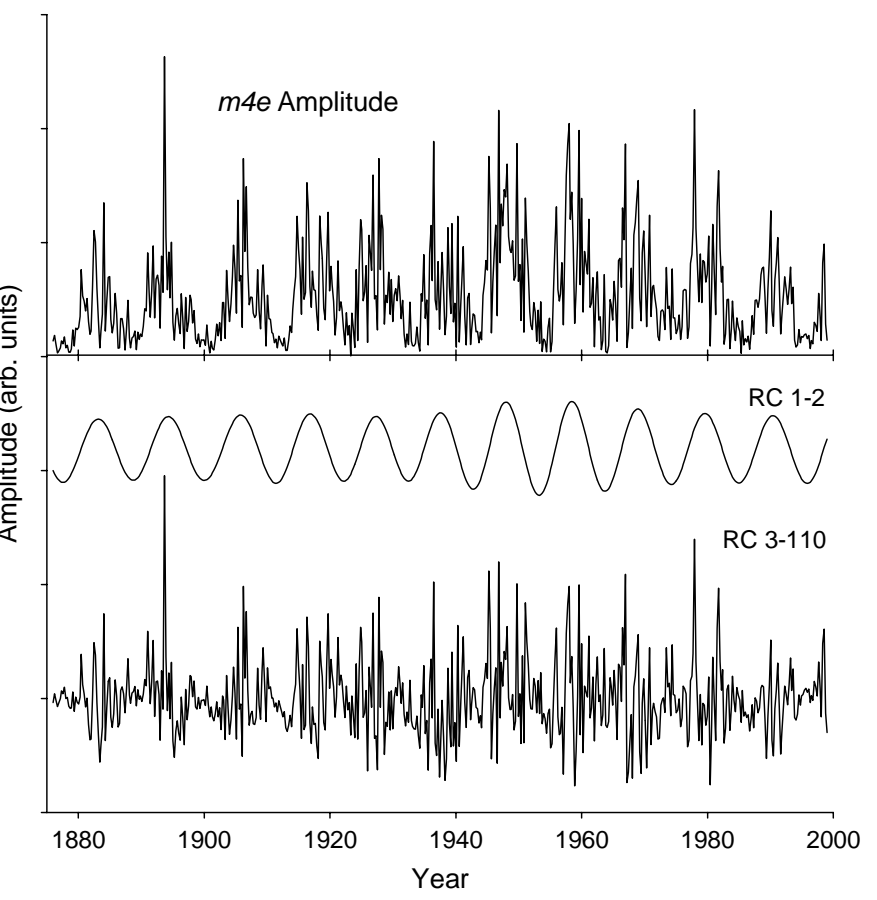

Fig. 12. Example of the amplitude series from $1 \mathrm{cSSH} m 4 e$ and SSA reconstructed series RC1-2; and RC3-110. The curves are of the same arbitrary amplitude scale but are vertically displaced for viewing.

must not only occur in many of the lcSSHs, but they must be persistent and stationary for the full 125 years of the data. Oscillations that are specific to individual solar cycles or to 

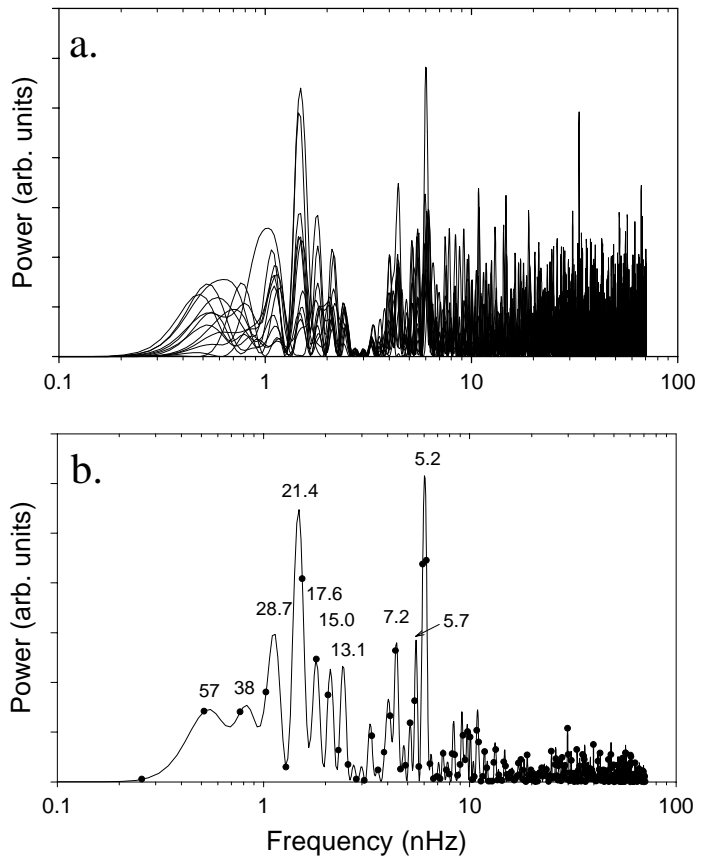

Fig. 13. Fourier transform spectra of RC3-110 amplitude series for individual and combined lcSSHs. a) Spectra of each symmetric lcSSH series up to order, 12. b) Spectra of the average RC3-110 series for the 12 symmetric lcSSHs. Computation of the spectrum, using no padding, yields the position and power of the harmonics of series length (dots). A better estimate of peak location, in the low frequency region, is clearly obtained with padding.

lcSSHs would tend to cancel in the summation series, even if they are not noise. Wavelet analysis was used on the summation series above $6 \mathrm{nHz}$ to determine if there are any oscillations that are common to the lcSSHS but specific to certain solar cycles. This is shown in Fig. 14. The third harmonic of the solar cycle, near $9.5 \mathrm{nHz}$ shows significant wavelet power when integrated over all years, but inspection of the 2D density plot indicates that each solar cycle tends to have a different frequency maximum near this value. Cycle-dependent oscillations of this nature, may indicate that each solar cycle represents an independent response to an impulse that repeats each $\sim 11$ years. A detailed wavelet analysis on each $1 \mathrm{cSSH}$ may yield additional evidence for this but is beyond the scope of this report.

The oscillations below $3 \mathrm{nHz}$, labeled in Fig. 13b, are very similar to the near-decadal oscillations appearing in various solar, geomagnetic, and cosmic ray series (Juckett 2001). With the exception of the 15-yr oscillation, they are nearly synchronous to the solar and geomagnetic series (not shown). The lcSSHs may offer a way to study the low intensity, non-11-yr oscillations that are sometimes strong in heliospheric and nearEarth phenomena driven by solar activity processes.

\section{Discussion}

\subsection{General comments}

The long, continuous record of sunspot group latitude and longitude positions offers the opportunity for a detailed look at the spatial dynamics of sunspot emergence on the solar surface.

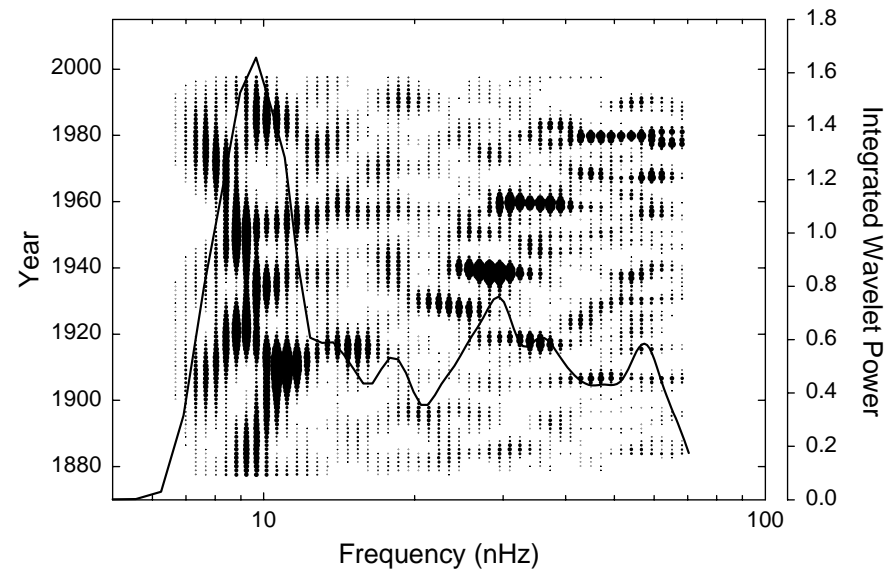

Fig. 14. Wavelet map and integrated power for the sum of the symmetric RC3-110 series in the frequency domain $>7 \mathrm{nHz}$. The average RC3-110 series was filtered by using the Fourier transform coefficients above $7 \mathrm{nHz}$ to reconstruct the time domain. Wavelet power is represented by a bubble plot and integrated power given as a solid line.

In this report, surface spherical harmonic decomposition, spanning the low harmonic orders 1-12, was performed on each of 1651 consecutive Carrington rotations. The temporal development of both the spatial phases and amplitudes of the SSHs were explored to extract torsional oscillations from phase and non-11-yr variations from amplitude series, respectively. While the $\mathrm{SSH}$ decomposition of each $\mathrm{CR}$ represents the distribution of individual sunspot groups, the long-term patterns in SSH phase and amplitude span many instances of sunspot emergence and, therefore describe the common regions of sunspot occurrence. Order and regularity at these time scales suggest long-term memory at the sites of flux tube release.

The SSH decomposition of each CR is, necessarily, performed on an estimate of the true sunspot distribution, as obtained from the progressively moving 50 percent observable surface of each rotation. The synoptic-style mapping is obtained by averaging the positions for each observed sunspot group. The mapping, however, is not weighted by area or duration. The lack of area involvement in simple clustering is shown in Fig. 3. Sunspot duration was not used because it can never be known adequately for many of the sunspots that move into the unseen hemisphere. Duration is also not a robust feature of sunspot patterns because sunspots may disappear and then reappear along the same cluster paths spanning many solar rotations. These instances of sunspot occurrence may be assigned different group numbers while potentially constituting a single emergence trend. In addition, the mapping necessarily excludes those short-lived sunspots that exist only on the backside. The result is a sampling of the total solar surface through the course of one $\mathrm{CR}$, but since the sampling is uniform and complete spatially, it is suitable for SSH decomposition. It precludes identification of temporal variations shorter than a single CR, but is adequate for detecting patterns spanning multiple CRs.

The focus of this report is on longitudinal variation. Whereas the SSHs offer a complete decomposition of the observable sunspot position patterns, the focus on longitude was realized by construction of symmetric and anti-symmetric 
combinations of constant $m$ value, generating sectoral lcSSHs. Analysis of these lcSSHs allowed a deeper examination of the patterning and temporal oscillations only hinted at in Fig. 2. This report was limited to the symmetric lcSSHs, but the antisymmetric lsSSHs show similar behavior in both spatial phase and amplitude variations. They also show some differences that may be associated with solar asymmetries. This will be addressed in a subsequent paper. The possible latitudinal preferences observed in Fig. 1 are not easily studied by these lcSSHs but may be addressed with SSH combinations of constant $l-m$. This, however, will also be the subject of future work.

\subsection{Phase drift and orbital torque}

The long-term phase stability seen in Fig. 8 indicates that there are very slowly changing processes that operate in sunspot genesis. The slight drift observed in Fig. 8 is very similar to the apparent retrograde drift in Fig. 2 over the same time frame. In Fig. 2 there also appears to be low order phase oscillations with time scales on the order of the solar cycle. Figures 7 and 8 reveal that such phase oscillations occur at several time scales and give the drift lines, seen in Fig. 8, a certain fuzzy thickness. The occasional phase shifts (inversions) in the drift lines of Fig. 8 indicate that other very long term modal oscillations are also occurring. The sources for many of these oscillations are unknown, but time series extracted from the phase-walk series suggest that Sun-Planet interactions may be the source of some of them.

The angular momentum of the solar system is conserved with respect to its center of mass. Due to the varying positions of the planets, the Sun wobbles around the center of mass with an apparently changing orbital angular momentum, $L(t)$. This implies that the balancing angular momentum changes occur in the remaining bodies of the solar system, but this has not been proven. Jose (1965) calculated $L(t)$ and interpreted the derivative $\mathrm{d} L / \mathrm{d} t$ as an orbital torque acting on the Sun caused by the varying relative positions of the giant planets. Since that time, several investigators have explored aspects of $L$ or $\mathrm{d} L / \mathrm{d} t$ with regard to solar cycle activity, sporadic energy releases of the Sun, and Earth's weather (e.g., Charvatova 1990, 1997; Fairbridge \& Sanders 1987; Landscheidt 1987, 1988, 1999). Since gravitationally based tidal effects are very weak for the distant giant planets, the explanation for any correlation between $\mathrm{d} L / \mathrm{d} t$ and these events relies on the existence of spin-orbit coupling between $L$ and the Sun's rotational momentum. This would introduce solar rotation into the orbital momentum balance equation. Such a coupling has not been modeled, because an appropriate mechanism has not been elucidated. Furthermore, the existence of any significant coupling has remained questionable because suitable direct evidence has been lacking. The torque-like oscillatory motions in the lcSSH spatial phases appear to offer the most direct evidence, to date, for the presence of significant spin-orbit coupling. It extends and corroborates the differential rotation analysis of Javaraiah \& Gokhale (1995), which revealed frequencies compatible with synodic conjunctions of some planets. They did not, however, examine $\mathrm{d} L / \mathrm{d} t$ or questions of synchrony.
Under the spin-orbit coupling assumption, it is expected that $\mathrm{d} L / \mathrm{d} t$ induces a corresponding counter torque operating in the spin frame and this is responsible for the phase shifts. A negative counter torque would induce retrograde motion (positive phase shift), therefore a positive $\mathrm{d} L / \mathrm{d} t$ should induce a positive phase shift, as seen. One might expect a lag of $\pi / 2$ between the two series, however, because one represents a force while the other a motion. This discrepancy is resolvable if latitudinal drifts are also considered because a momentumaltering force should induce a Coriolis-type rotation, which would have maximal longitudinal displacement at the peak of the torque. Latitudinal drifts, however, are more difficult to detect in the sectoral lcSSHs, so this speculation awaits further investigation.

\subsection{Non-11-yr amplitude oscillations}

The non-11-yr oscillations in the average amplitude series of the lcSSHs are divided into two frequency ranges bracketing the $3 \mathrm{nHz}$ solar cycle region. Many of these oscillations can also be extracted from the monthly Wolf sunspot series, but their significance has been questionable due to their low power in that series. The appearance of these oscillations in each of the lcSSHs generates a higher probability that they are real components of sunspot variation and creates the possibility of understanding their origins.

The two strongest components are the 21.4-yr and the 5.2-yr oscillations. The first is the expected Hale cycle frequency and the second is probably the 2nd harmonic of the $11-y r$ solar cycle. The Hale cycle is defined by magnetic polarity alternations in sunspot pairs and in the solar dipolar field, and may represent the fundamental solar cycle. Its presence in the Wolf series and in SSH amplitudes probably results from alternating peak heights in the solar cycle because it is shifted by $\pi / 2$ with respect to the magnetic Hale cycle (Juckett 2001). An oscillation near the 2 nd harmonic tends to give the Wolf series an asymmetric shape and may be responsible for the difference in rise and fall times in each cycle. Its presence is also consistent with the hypothesis that the Sun is a resonant body with dynamics governed by non-linear excitatory and restoring forces generating multiple harmonics. The presence of a cycle-specific third harmonic, near $9 \mathrm{nHz}$, further supports this general notion.

The cluster of frequencies near the 21.4-yr oscillation resembles a pattern that might be generated by the related processes of frequency modulation and non-linear mixing of waveforms. Several of these frequencies are seen in geomagnetic, cosmic ray, and other solar series but their origin, like the fundamental solar cycle, is unknown. In a previous speculative report (Juckett 2000), the non-linear mixing of solar orbital momentum, $L(t)$, and a spin projection function, $P(t)$, generated eight frequencies from the splitting of the 19.6-yr $L(t)$ fundamental by the orbital periods of each of the four giant planets. The resulting frequencies have the following periods; $7.4,11.9$, $16.0,17.8,22.7,26.1,29.1$, and $61.6 \mathrm{yr}$. This pattern is very similar to that in Fig. 13 and may offer additional evidence for a role of the Sun's orbital momentum, $L$, in sunspot variation. 
An examination of Fig. 7 reveals that the high frequency oscillations, indicated by the $\pi$-phase jumps, have periods on the order of $0.5-2.0$ years and these vary significantly with time. Unlike the frequencies of Fig. 13, which are common to all lcSSHs, these rapid oscillations are different for each lcSSH and for each solar cycle. Further analysis will require the dissection of noise from the signal plus the ability to treat each cycle and each harmonic separately. A preliminary analysis of selected cycles (not shown) suggests that a wave packet model may provide a useful tool for studying these rapid oscillations, provided the instantaneous frequency of the packet cycles is inversely correlated to amplitude.

\subsection{Conclusion}

A surface spherical harmonic analysis has been performed on sunspot group distributions for each of 1625 Carrington rotations. A few, selected results have been presented to highlight the variations that occur in sunspot longitudinal patterns. The time series generated from spatial phase and harmonic amplitude offer different types of information on sunspot variation. The amplitudes give a more detailed presentation of the variation in sunspot prevalence throughout this era than is obtainable from the Wolf sunspot series. The spatial phases detect torsional motion in sunspot modal patterns and offer evidence for spin-orbit coupling in the Sun's dynamic.

Acknowledgements. I am grateful for the support and encouragement of Dr. Barnett Rosenberg. This work was supported by a joint research agreement between Barros Research Institute and Michigan State University.

\section{References}

Benevolenskaya, E. E., Hoeksema, J. T., Kosovichev, A. G., \& Scherrer, P. H. 1999, ApJ, 517, L163

Berger, A., Melice, J. L., \& vanderMersch, I. 1990, Phil. Trans. R. Soc. Lond. A, 330, 529
Bogart, R. S. 1982, Sol. Phys., 76, 155

Charvatova, I. 1990, Bull. Astron. Inst. Czechosl., 41, 200

Charvatova, I. 1997, Surveys in Geophys., 18, 131

Das, T. K., \& Nag, T. K. 1999, Sol. Phys., 187, 177

De Toma, G., White, O. R., \& Harvey, K. L. 2000, ApJ, 529, 1101

Erofeev, D. V. 1999, Sol. Phys., 186, 431

Erofeev, D. V. 2001, Sol. Phys., 198, 31

Fairbridge, R. W., \& Sanders, J. E. 1987, in Climate, ed. M. R. Rampino, J. E. Sanders, W. S. Newman, \& L. K. Konigsson, (Van Nonstrand Reinhold, New York), 446

Feynman, J., \& Gabriel, S. B. 1990, Sol. Phys., 127, 393

Gokhale, M. H., \& Javaraiah, J. 1990, MNRAS, 243, 241

Gokhale, M. H., \& Javaraiah, J. 1995, Sol. Phys., 156, 157

Gokhale, M. H., Javaraiah, J., Narayanan Kutty, K., \& Varghese, B. A. 1992, Sol. Phys., 138, 35

Howard, R. F. 1996, ARA\&A, 34, 75

Javaraiah, J., \& Gokhale, M. H. 1995, Sol. Phys., 158, 173

Javaraiah, J., \& Gokhale, M. H. 1997, Sol. Phys., 170, 389

Javaraiah, J., \& Komm, R. W. 1999, Sol. Phys., 184, 41

Jose, P. D. 1965, AJ, 70, 193

Juckett, D. A. 1998, Sol. Phys., 183, 201

Juckett, D. A. 2000, Sol. Phys., 191, 201

Juckett, D. A. 2001, J. Geoph. Res., 106, 18651

Landscheidt, T. 1987, Sol. Phys., 101, 195

Landscheidt, T. 1988, Climate Change, 12, 265

Landscheidt, T. 1999, Sol. Phys., 189, 415

Lean, J. L., \& Brueckner, G. E. Q. 1989, ApJ, 337, 568

Mikhailutsa, V. 1995, Sol. Phys., 159, 29

Mikhailutsa, V., \& Makarova, V. V. 1994, Sol. Phys., 155, 391

Mikhailutsa, V., \& Makarova, V. V. 1999, Astron. Astroph. Trans., 17, 393

Petrovay, K., \& Abuzeid, B. K. 1991, Sol. Phys., 131, 231

Rivin, Y. R. 1999, Sol. Phys., 187, 207

Simon, P. A., \& Legrand, J. P. 1992, Sol. Phys., 141, 391

Stenflo, J. O., \& Vogel, M. 1986, Nature, 319, 285

Torrence, C., \& Campo, G. 1998, Bull. Am. Meteor. Soc., 79, 61

Uddin, W., Pande, M. C., \& Verma, V. K. 1991, Ap\&SS, 181, 111

Vautard, R., Yiou, P., \& Ghil, M. 1992, Physica D, 58, 95

Verma, V. K., Joshi, G. C., \& Paliwal, D. C. 1992, Sol. Phys., 138, 205

Wolff, C. L. 1983, ApJ, 264, 667 